\title{
Slow halo CMEs with shock signatures ${ }^{\star}$
}

\author{
S. Pohjolainen ${ }^{1,2}$ and N. J. Lehtinen ${ }^{1}$ \\ 1 Tuorla Observatory, University of Turku, Väisäläntie 20, 21500 Piikkiö, Finland \\ e-mail: [silpoh;nijole]@utu.fi \\ 2 Department of Physics, University of Turku, Finland
}

Received 30 August 2005 / Accepted 17 November 2005

\section{ABSTRACT}

Context. In the solar corona, shocks are formed when the speed of a disturbance exceeds the local magnetosonic speed. In the active region corona the Alfvén speed can drop to a few hundred $\mathrm{km} \mathrm{s}^{-1}$, but globally it is much higher. There has been a long debate on whether the shocks responsible for type II bursts are created by bow shocks in front of coronal mass ejections (CMEs), shocks in the flanks of CMEs, or by flare (blast) waves.

Aims. We study the alternative explanations for type II bursts in events where we have a slow CME, flare(s), and associated type II burst emission.

Methods. We use multi-wavelength observations to analyse the height-time evolution of CMEs and compare it with the evolution of shock signatures in radio and EUV.

Results. Three flare-associated halo-type CME events were observed on October 30, 2004. Velocity estimates $\left(260,325\right.$, and $\left.920 \mathrm{~km} \mathrm{~s}^{-1}\right)$ from the first plane-of-the-sky CME leading front observations suggested that the first two were very slow compared to halo CMEs, on average. The CMEs were associated with flares (M 4.2, X1.2, and M 5.9) and each event was also associated with coronal (metric) type II emission that is known to be a signature of a propagating shock front. After the flare starts, loop displacements and large-scale dimmings were observed in EUV. The two slow halo CMEs started as filament eruptions, but the CME velocities and/or bulk motions were affected at the times of flares. We find support for the idea that the cause of metric type II bursts in these two events is flare-related. The later CME velocity changes (acceleration around 4-5 solar radii) could also be explained by eruptions associated with later flares. The repeating homologous flare-halo CME events indicate a restoration of the same large-scale structures within 5-6 h.

Key words. Sun: flares - Sun: filaments - shock waves - Sun: coronal mass ejections (CMEs) - Sun: radio radiation

\section{Introduction}

Coronal mass ejections (CMEs) are energy release processes that can lead to a significant expulsion of mass from the Sun. However, it is still poorly understood how CMEs are formed. The relationship between CMEs, flares, type II radio bursts and wave phenomena has been under debate for a long time. A good timing agreement has been found between the impulsive phases of flares and metric type II bursts (e.g., Harvey 1965; Vrs̆nak et al. 1995; Klassen et al. 1999, 2003), as well as between Moreton waves and type IIs (e.g., Hudson et al. 2003; Warmuth et al. 2004a,b, and references therein). There is also quite good synchronization between CME acceleration and the impulsive phases of flares (Zhang et al. 2001, 2004; Vršnak et al. 2004, and references therein). Because of these, it is often difficult to decide whether a coronal shock (indicated by a metric type II burst) is ignited by a flare or if it is driven by a CME.

* Magnetic field movie is only available in electronic form at http://www.edpsciences.org and at http://www. astro.utu.fi/tuorla/solar/30oct2004/
Wu et al. (2004) have suggested that different types of initiation processes in a "standard" prominence configuration could determine if a CME becomes fast or slow, and the fast flare/slow non-flare CME classification has also been recently questioned by Vrs̆nak et al. (2005). Massive velocity changes and exponential growth in the CME speed has been suggested to explain the large speed differences between the rising flux ropes at low altitudes and the later observed CME fronts (Vrs̆nak 2001a; Shanmugaraju et al. 2003; Gallagher et al. 2003, and references therein). In some cases the obtained kinematic curves can be quite peculiar (Goff et al. 2005).

"Halo" type CMEs associated with flares are of special interest since their signatures can be observed directly on the disk (Zarro et al. 1999; Webb et al. 2000; Pohjolainen et al. 2001; Harra \& Sterling 2001; Jing et al. 2004; Pohjolainen et al. 2005). Sometimes homologous CME/flare events happen in close succession, and then questions arise on their trigger mechanism and on how the field can restore itself on short time scales (Chertok et al. 2004; Zhang \& Wang 2002: homologous CMEs 7-10 h apart; Moon et al. 2002: homologous flares less 
than one hour apart). Halo CMEs are known to be very fast, the measured apparent speeds are $957 \mathrm{~km} \mathrm{~s}^{-1}$ on average (Yashiro et al. 2004), which is twice the speed of "normal" CMEs. Speed estimation can, however, be difficult (see Michałek et al. 2003), as CMEs are observed as scattered light on the plane of the sky. Using both white light and radio observations the "true" speeds have in some studies been estimated to be higher than the measured plane-of-sky speeds (Reiner et al. 2003).

Solar radio type II bursts are thought to be caused by MHD shock waves propagating through the corona and interplanetary medium (e.g. Nelson \& Melrose 1985). Both flare (blast) waves and piston-driven bow shocks could be the drivers. There is discussion of whether coronal metric type II bursts and interplanetary decametric-hectometric (DH) type II bursts are related at all (see, e.g., Cane \& Erickson 2005; Mancuso \& Raymond 2004; Reiner et al. 2001). The usual metric type II burst frequency drifts are in the range of $0.1-1.0 \mathrm{MHz} \mathrm{s}^{-1}$, and the derived burst exciter speeds have been estimated to be in the range of 400-2000 km s${ }^{-1}$ (Nelson \& Melrose 1985). The wide speed range mainly reflects the problem of transforming frequency drifts into speeds using different atmospheric density models, especially at low atmospheric heights.

Only a small number of flares and CMEs are accompanied by type II bursts, but almost all type II bursts are associated with flares and CMEs (see, e.g., Cliver et al. 1999). Flare characteristics and type II burst parameters have shown good correlation (see, e.g., Vršnak 2001b). A good correlation has also been found between the CME speeds and the inferred speeds of DH type II bursts, contrary to metric type IIs (Reiner et al. 2001). Furthermore, CMEs associated with DH type II bursts are found to be generally faster and wider than those associated with metric type II bursts (Gopalswamy et al. 2000; Lara et al. 2003). A "timing problem" has been observed between CMEs and type II bursts, as CME launch time estimates are much earlier than the associated type II onset times (Cliver et al. 2005). Cliver et al. suggest that rapid CME acceleration could still act as the principal driver for metric type II bursts instead of flare blast waves/ejecta. The leading edges of filament-associated CMEs accelerate gradually, to speeds typically in the range of 400-600 $\mathrm{km} \mathrm{s}^{-1}$ (Sheeley et al. 1999). Observations of accelerating filaments at the time of an associated flare have been reported by, e.g., Qiu et al. (2001), Kundu et al. (2004), and Sterling \& Moore (2005), but in these cases the velocity change happens at low heights when the flare site and the filament are separated by less than $\sim 0.5 R_{\odot}$.

This paper presents analysis on the relationship between CMEs, flares, and type II bursts in three halo-type CME events that originated from the same active region only 5-6 h apart on the same day. Sections 2 and 3 describe the observed characteristics and in Sect. 4 we present a summary. Results and discussion are presented in Sect. 5.

\section{Repeated flare-halo CMEs on October 30, 2004}

Three halo-type CMEs were recorded by the Solar and Heliospheric Observatory (SOHO) Large Angle and Spectrometric Coronagraph (LASCO) experiment (Brueckner et al. 1995) on October 30, 2004. The halo CMEs showed
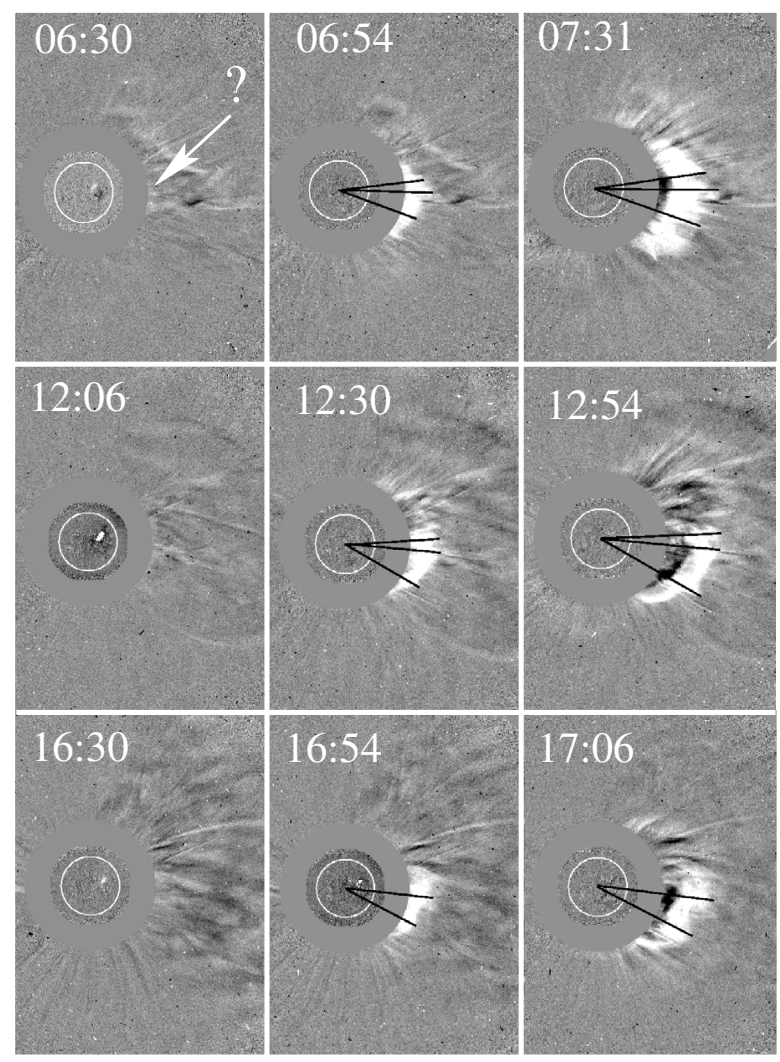

Fig. 1. The first appearances of the three homologous-looking halo CMEs with brightness asymmetry on October 30, 2004. The CMEs were first detected by LASCO C2 at 06:54 UT, 12:30 UT, and 16:54 UT. The images show our method of estimating the CME speeds: the distances from the Sun center to the front locations were measured along several different radials. Radials were selected when the front showed distinctive features that could be identified in the next LASCO image.

clear brightness asymmetry: the heliocentric distance of each CME was roughly the same in all directions, but the CME brightness was significantly different. In the first LASCO images all the halo-type CMEs had the appearance of a bright and wide ragged loop front over the West limb, see Fig. 1 and the data in the LASCO CME Catalog at http://cdaw.gsfc.nasa.gov/CME_list/. According to the Catalog, the halo CMEs were first observed at 06:54 UT (plane-of-the-sky leading front at heliocentric height $3.3 R_{\odot}$ ), at 12:30 (leading front at $3.2 R_{\odot}$ ), and at 16:54 UT (leading front at $\left.3.0 R_{\odot}\right)$. The estimated velocities from the leading front locations using the first two measurements of each event, 06:54-07:31 UT, 12:30-12:54 UT, and 16:54-17:06 UT, were $260 \mathrm{~km} \mathrm{~s}^{-1}, 325 \mathrm{~km} \mathrm{~s}^{-1}$ and $920 \mathrm{~km} \mathrm{~s}^{-1}$, respectively. The halo-type CMEs are listed in Table 1 as "Halo1", "Halo2", and "Halo3".

A slow (estimated speed $130 \mathrm{~km} \mathrm{~s}^{-1}$ ) faint CME had lifted off well before Halo1, it is listed as "CME1" in Table 1. The CME1 propagated towards the West, and it had reached a height of $\sim 5 R_{\odot}$ at the time of Halo1 detection. The height-time profiles of CME1 and Halo1 indicate that the leading front of 
Table 1. Properties of the CME-related events on October 30, 2004.

\begin{tabular}{|c|c|c|c|c|c|c|c|c|c|}
\hline Event & $\begin{array}{c}\mathrm{CME} \\
\text { speed }^{a} \\
\left(\mathrm{~km} \mathrm{~s}^{-1}\right)\end{array}$ & $\begin{array}{l}\text { Feature } \\
\text { speed }^{b} \\
\left(\mathrm{~km} \mathrm{~s}^{-1}\right)\end{array}$ & $\begin{array}{c}\text { CME } \\
\text { start }^{c} \\
(\mathrm{UT})\end{array}$ & $\begin{array}{c}\text { Type II } \\
\text { start } \\
\text { (UT) }\end{array}$ & $\begin{array}{r}\text { Type II } \\
\text { start } \\
\text { (F-lane) }\end{array}$ & $\begin{array}{c}\text { EIT } \\
\text { dimming } \\
\text { (UT) }\end{array}$ & $\begin{array}{c}\text { GOES } \\
\text { flare } \\
\text { Class }\end{array}$ & $\begin{array}{r}\text { Flare } \\
\text { start } \\
\text { (UT) }\end{array}$ & Notes \\
\hline \multirow[t]{2}{*}{ CME1 } & 130 & & $>00: 00$ & $00: 48$ & $70 \mathrm{MHz}$ & & C9.7 & $00: 39$ & Faint CME, only 3 points \\
\hline & & & & $03: 32$ & $100 \mathrm{MHz}$ & & M3.3 & $03: 23$ & \\
\hline \multirow[t]{3}{*}{ Halo1 } & 260 & $210 / 335$ & $05: 30$ & $\begin{array}{l}06: 15 \\
06: 42\end{array}$ & $\begin{array}{c}150 \mathrm{MHz} \\
3.2 \mathrm{MHz}\end{array}$ & 06:12 & M 4.2 & 06:04 & \multirow[t]{3}{*}{$\begin{array}{l}\text { CME acceleration/velocity change } \\
\text { around 08:30 UT near } 5 R_{\odot}\end{array}$} \\
\hline & & & & 07:03 & $90 \mathrm{MHz}$ & $07: 13$ & $\mathrm{C} 5.5$ & $06: 54$ & \\
\hline & & & & $07: 32$ & $10 \mathrm{MHz}$ & & & & \\
\hline CME2 & 990 & & 09:30 & 09:29 & $120 \mathrm{MHz}$ & $09: 36$ & M 3.7 & 09:09 & CME decelerates \\
\hline \multirow[t]{2}{*}{ Halo2 } & 325 & $355 / 550$ & $11: 35$ & $\begin{array}{l}11: 45 \\
11: 45\end{array}$ & $\begin{array}{r}140 \mathrm{MHz} \\
80 \mathrm{MHz}\end{array}$ & $11: 36$ & $\mathrm{X} 1.2$ & $11: 36$ & $\begin{array}{l}\text { CME acceleration/velocity change } \\
\text { around 13:30 UT near } 5 R_{\odot}\end{array}$ \\
\hline & & & & $11: 58$ & $44 \mathrm{MHz}$ & & & & \\
\hline CME3 & - & 340 & $14: 05$ & - & & - & $\mathrm{C} 2.6$ & $<14: 40$ & CME not catalogued, faint \\
\hline \multirow[t]{2}{*}{ Halo3 } & 920 & $810 / 900$ & $16: 25$ & $16: 30$ & $65 \mathrm{MHz}$ & $16: 24$ & M 5.9 & $16: 14$ & CME decelerates \\
\hline & & & & $16: 44$ & $14 \mathrm{MHz}$ & & & & \\
\hline
\end{tabular}

${ }^{a}$ Plane-of-the-sky speed of the leading front, from the first two available height-time data points (CME Catalog, Catholic Univ. of America).

${ }^{b}$ Our speed estimate from the identified CME features in the first 2-4 LASCO C2 images (minimum/maximum).

${ }^{c}$ Extrapolated backwards to the AR location, linear fit to front features/leading edge projected height.

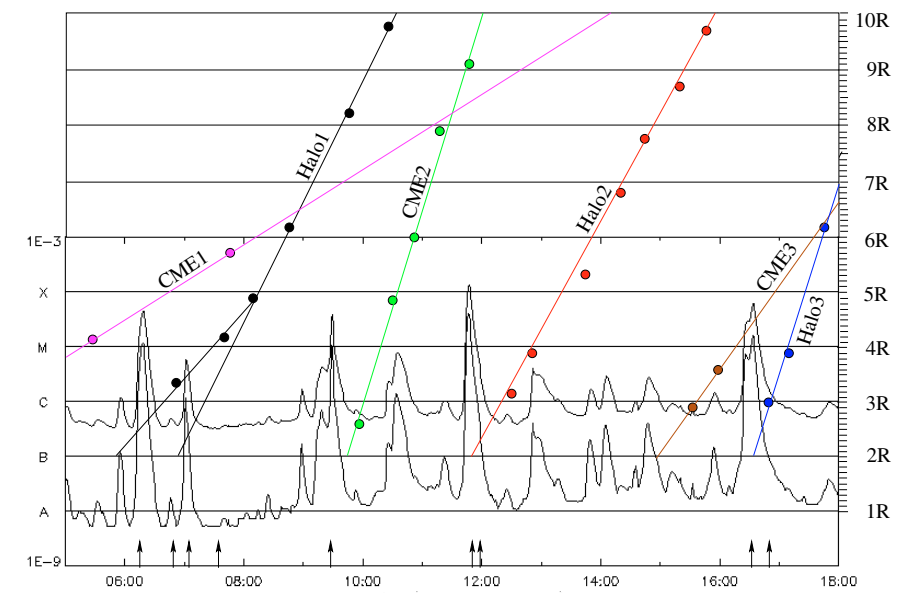

Fig. 2. GOES soft X-ray flux curve at $05-18$ UT with LASCO CME Catalog CME height-times. The CME heights in the Catalog are measured from the plane-of-the-sky outermost bright feature and no projection effects are taken into account. Linear fits to all data points can give erratic results on the start times, see e.g. the two different heighttime fits for Halo1. Black arrows mark the start times of type II radio bursts.

Halo1 would overtake the CME1 front around 09:00 UT near $6.5 R_{\odot}$, see Fig. 2 .

In between Halo1 and Halo2 a fast CME ("CME2") originated from the same active region with a plane-of-the-sky leading front velocity of $990 \mathrm{~km} \mathrm{~s}^{-1}$ (09:54-10:34 UT). CME2 also propagated towards the West, but it had a narrow dome-like structure instead of a bright loop. Because of the fast speed and timing (about three hours after Halol and more than two hours before Halo2), there is no indication of CME interaction at coronal heights.

Before the detection of the Halo3 front at 16:54 UT, a faint CME structure ("CME3") was observed in the LASCO images at 15:30 and 15:54 UT. This event is not listed in the
LASCO CME Catalog and we could only identify a bright front at 15:30 UT. Behind this front some dark structures can be identified in both images, and the projected speed of these is estimated to be around $340 \mathrm{~km} \mathrm{~s}^{-1}$. A linear fit to the heights of these structures suggests that CME3 and Halo3 reached the same height around 17:30 UT near $6 R_{\odot}$, see Fig. 2.

Several flares occurred in NOAA AR 10691, located at N13W20 at the beginning of the analysed time period (05-18 UT). The first CME ("CME1") that had started before the analysed time period could have had association with at least two flares, a C9.7 class flare starting at 00:39 UT and a M 3.3 class flare starting at 03:23 UT, but these will be excluded from this analysis although they are listed in Table 1.

Two flares were observed in association with Halo1, the first started at 06:04 UT (GOES class M 4.2) and the second one at 06:54 UT (class C5.5). Both GOES flares showed a simple one-peak structure. The following CME2 could be associated with a class M3.7 flare that had started at 09:09 UT, but it showed a double-peak structure where the flux started to rise again at 09:24 UT. Halo2 was associated with a onepeak, class X1.2 flare that started at 11:36 UT. The following CME3, being faint and slow, is difficult to associate with any one flare, but several C-class flares occurred between 13:30 and 15:00 UT near the time of the estimated CME3 "lift-off". Halo3 was associated with a class M 5.9 flare that started at 16:12 UT and had a double-peak structure; the second flux rise started at 16:30 UT.

Halo1 and Halo2 events were imaged in $\mathrm{H} \alpha$ by Yunnan in China and Kanzelhöhe in Austria (Global High Resolution $\mathrm{H} \alpha$ Network Observatories). In both events filament eruptions are observed before the X-ray flares, see Fig. 3. A very bright arclike pattern also formed during both flares.

Full disk EUV images, taken by the EUV Imaging Telescope (EIT) on board $\mathrm{SOHO}$, are available throughout the analysed time period with a 12 min image cadence on average 

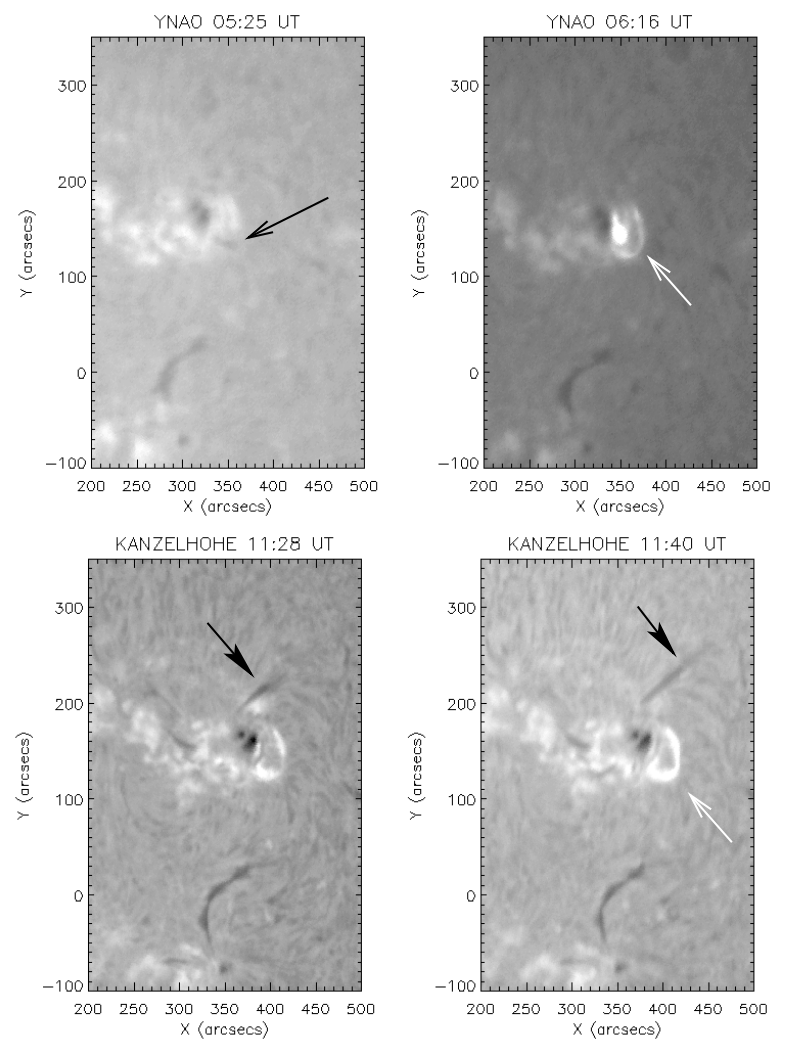

Fig. 3. $\mathrm{H} \alpha$ flares recorded by Yunnan in China (first event, upper row) and by Kanzelhöhe in Austria (second event, bottom row). Black arrows point to the erupting filaments, and white arrows to the flaring bright arc-like structures. The $\mathrm{H} \alpha$ feature in the second event can be the leg of an erupting filament, and therefore the filament summit could be considerably higher. Observations at both sites were affected by changing weather conditions.

(Delaboudinière et al. 1995). The 195 Å running difference images show that all the halo CME events were associated with homologous-looking, large-scale EUV dimmings, see Fig. 4. The dimmings first appeared at nearby loop footpoints and then spread towards the Southwest. The difference images also show how some of the large loops were displaced, i.e. that they were moving. A displaced or temperature changing loop was also observed in association with the post-Halo1 flare (Fig. 5). The faint dimmings in the Southwest at that time could still be due to the preceding Halo1. The CME2 that occurred in between Halo1 and Halo2 was associated with a narrow dimming in the Southwest, but the main part of the CME-associated eruption was directed towards the Nortwest, Fig. 5. The flare start times and the EUV dimming start times are listed in Table 1.

High resolution line-of-sight magnetic field observations provided by the $\mathrm{SOHO}$ Michelson Doppler Imager (MDI, Scherrer et al. 1995) show a growing, mainly positive polarity region near N13W20. The active region was connected to a smaller, mainly negative polarity region near S01W17 (NOAA AR 10690). The magnetic flux evolution of these two regions is shown in Fig. 6, and the spatial changes can be viewed in the magnetogram movie at http://www .astro.utu.fi/tuorla/solar/30oct2004/ and at http://www. edpsciences.org.
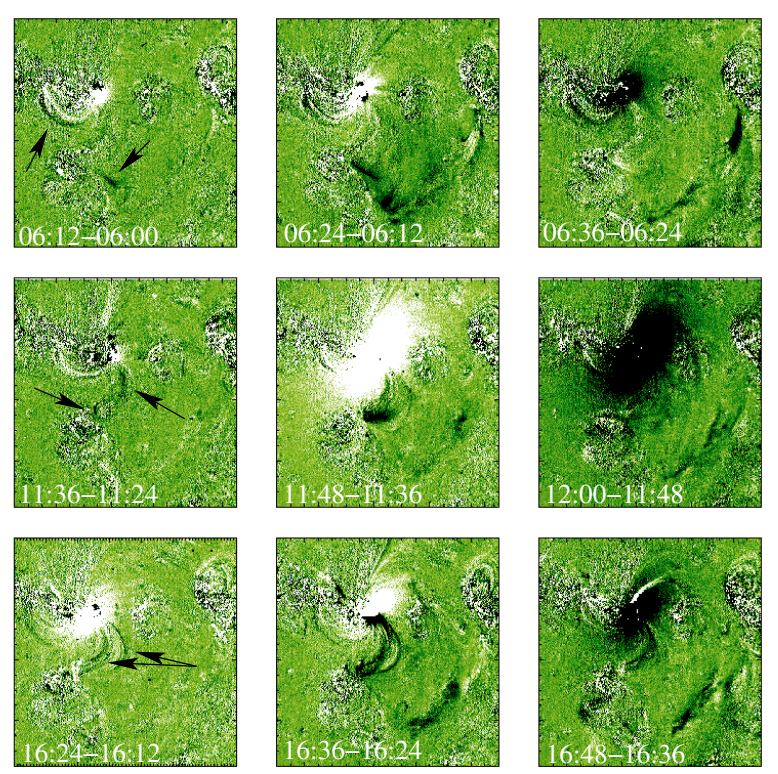

Fig. 4. SOHO EIT $195 \AA$ A running difference images from the times of the three halo CMEs. The shown area is $800^{\prime \prime} \times 750^{\prime \prime}$ in each frame. Arrows point to the displaced loop systems and regions that dimmed first. Note that the similar looking large-scale dimmings were all directed towards the Southwest.
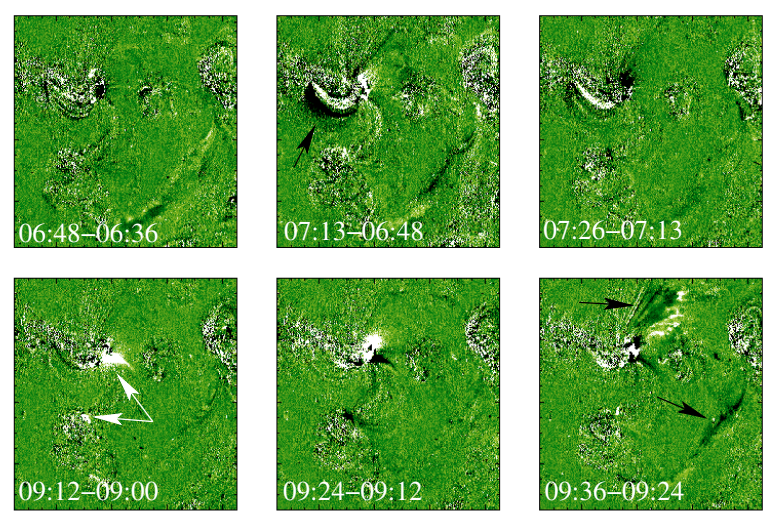

Fig. 5. SOHO EIT running difference images from the times of the post-Halo1 flare and CME2. The shown area is $800^{\prime \prime} \times 750^{\prime \prime}$, as in Fig. 4. The dimmings at 06:48 UT are due to the preceding Halo1 event. The flare started at 06:54 UT and a change in the active region loop is visible at 07:13 UT (black arrow). The flare associated with CME2 started at 09:09 UT and a flare brightening is visible in the AR and in a region south of it (white arrows). This looks to be a possible case of sympathetic flaring. An eruption towards the Northwest is visible at 09:36 UT, as well as a dimming in the Southwest.

\section{Shock signatures in radio emission}

The full time period under analysis could be covered with spectral observations in the plasma frequency domain at radio wavelengths. Observations were provided by HiRAS/Hiraiso Solar Observatory in Japan (25-2500 MHz), IZMIRAN/Solar Radio Laboratory in Russia (25-270 MHz), OSRA/AIP in Germany (40-800 MHz), Phoenix-2/ETHZ in Switzerland $(112-3970 \mathrm{MHz}), \mathrm{DAM} /$ Observatoire de Paris in France (20-70 MHz), GBSRBS/NRAO in USA (18-70 MHz), and by the WAVES experiment on the WIND spacecraft (RAD2 

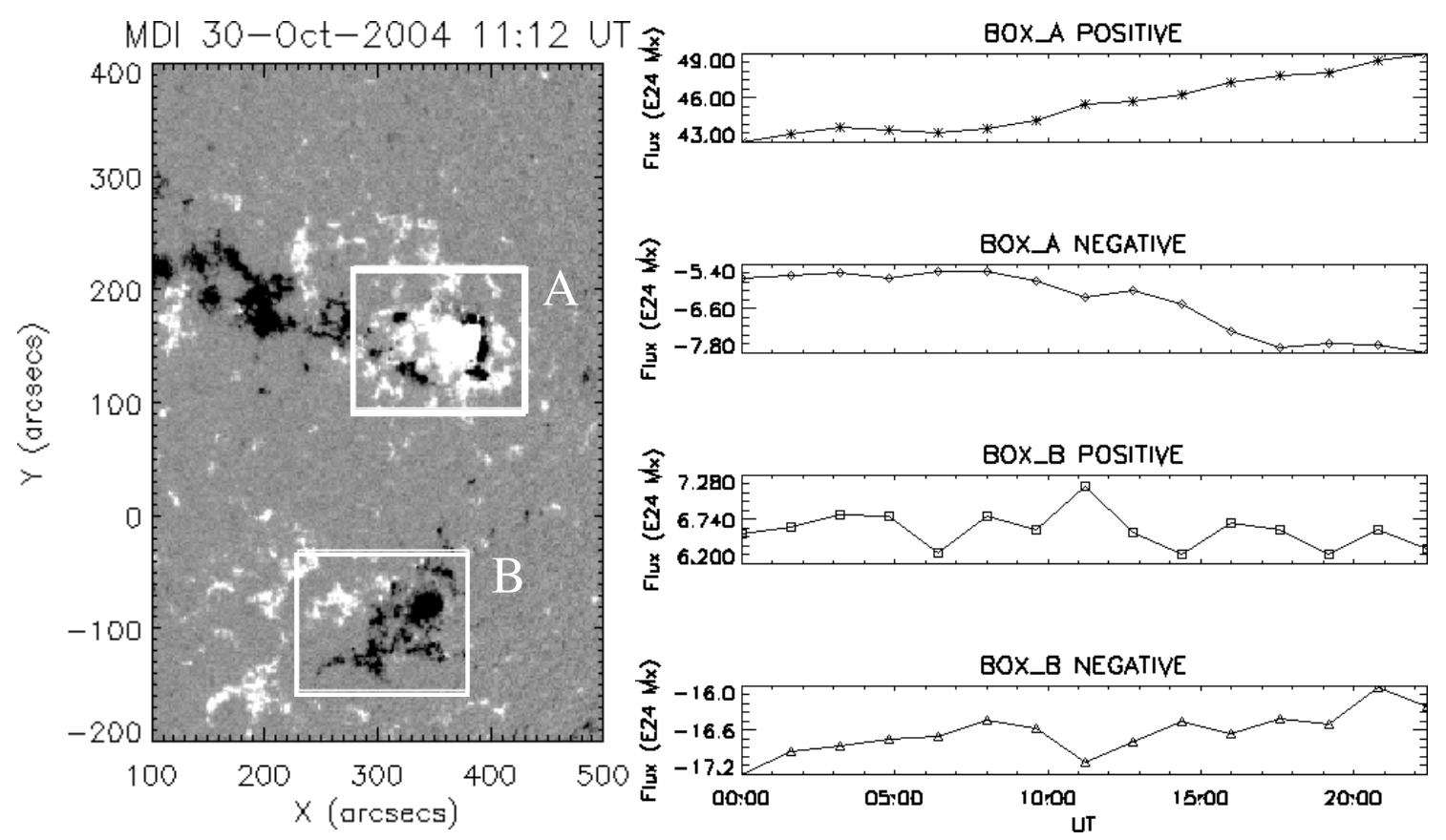

Fig. 6. Boxed areas show the flaring active region A and the interconnecting region B in the SOHO MDI magnetogram at 11:12 UT. The magnetic flux evolution for both regions (positive and negative, in $10^{24} \mathrm{Mx}$ ) is shown on the right.
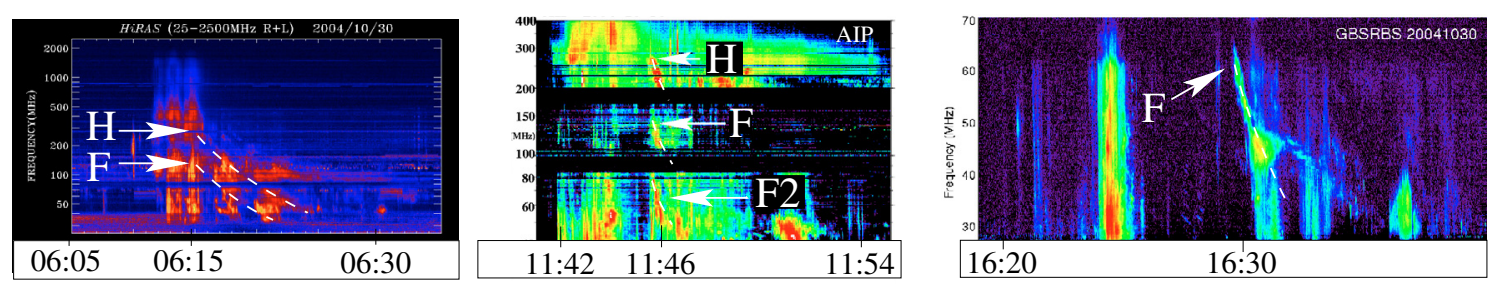

Fig. 7. Decimetric-metric type II bursts recorded by HiRAS, AIP OSRA, and GBSRBS in association with the three flare-halo CME events. Flare-associated radio emission started at 06:12, 11:42, and 16:24 UT, respectively. The type II burst start times are at 06:15 UT (150 MHz fundamental emission, second harmonic visible at $300 \mathrm{MHz}$ ), 11:45 UT (140 MHz fundamental emission, second harmonic at $280 \mathrm{MHz}$, a possible second type II burst fundamental emission lane starting at $80 \mathrm{MHz}$, second harmonic mixing with the fundamental lane of the other burst), and at 16:30 UT (65 MHz fundamental emission, possibly band-split).

observations at $1-14 \mathrm{MHz})$. Most of these data are available from each observatory/experiment Webpage.

All three halo-type CME events were associated with strong metric type II burst emission, as were some of the flares/CMEs before and in between the halos, see Table 1. Figure 7 shows the dynamic radio spectra from the Hiraiso Solar Observatory (Halo1 event), AIP Tremsdorf (Halo2 event) and Green Bank (Halo3 event). The HiRAS spectrum shows both the fundamental $(\mathrm{F})$ and second harmonic $(\mathrm{H})$ emission lanes, with the F-lane starting at $150 \mathrm{MHz}$. The AIP spectrum also shows $\mathrm{F}$ and $\mathrm{H}$ emission (F-lane starting around $140 \mathrm{MHz}$ ), but there is indication of another emission source at $80 \mathrm{MHz}$ ("F2") where the second harmonic is mixed with the F-lane of the first burst. The GBSRBS spectrum shows only one emission lane starting around $65 \mathrm{MHz}$. The measured drift rates were $0.6 \mathrm{MHz} \mathrm{s}^{-1}, 2.1 \mathrm{MHz} \mathrm{s}^{-1}$ and $0.4 \mathrm{MHz} \mathrm{s}^{-1}$, respectively. The drift rate of the second type II burst is larger than normally seen in type II events, average drifts being in the
$0.1-1.0 \mathrm{MHz} \mathrm{s}^{-1}$ range. The fast-drifting emission sources are visible only for about one minute, and slower-drift sources appear in the spectrum later, observed by IZMIRAN and DAM.

The WAVES RAD2 measurements from the Wind satellite (Bougeret et al. 1995) were checked for interplanetary type II burst emission at decametric-hectometric (DH) wavelengths. Only the first event was clearly associated with DH type II burst emission, see Fig. 8. A type II lane was observed in the spectrum around $3 \mathrm{MHz}$ at 06:45 UT, indicated by the dashed line in Fig. 8. The RAD2 spectrum also shows a pair of type II burst lanes, starting at 07:29 UT at $10 \mathrm{MHz}$ (lanes indicated by dashed-dotted lines in Fig. 8). A possible continuation of the metric type II burst emission associated with the Halo3 event is visible at 16:44 UT near $14 \mathrm{MHz}$. All the observed type II bursts with start times and frequencies are listed in Table 1.

As the radio emission is generated via the plasma emission mechanism, the burst frequency $f(\mathrm{~Hz})$ of radio observations directly measures the plasma electron density $n_{\mathrm{e}}\left(\mathrm{cm}^{-3}\right)$ 


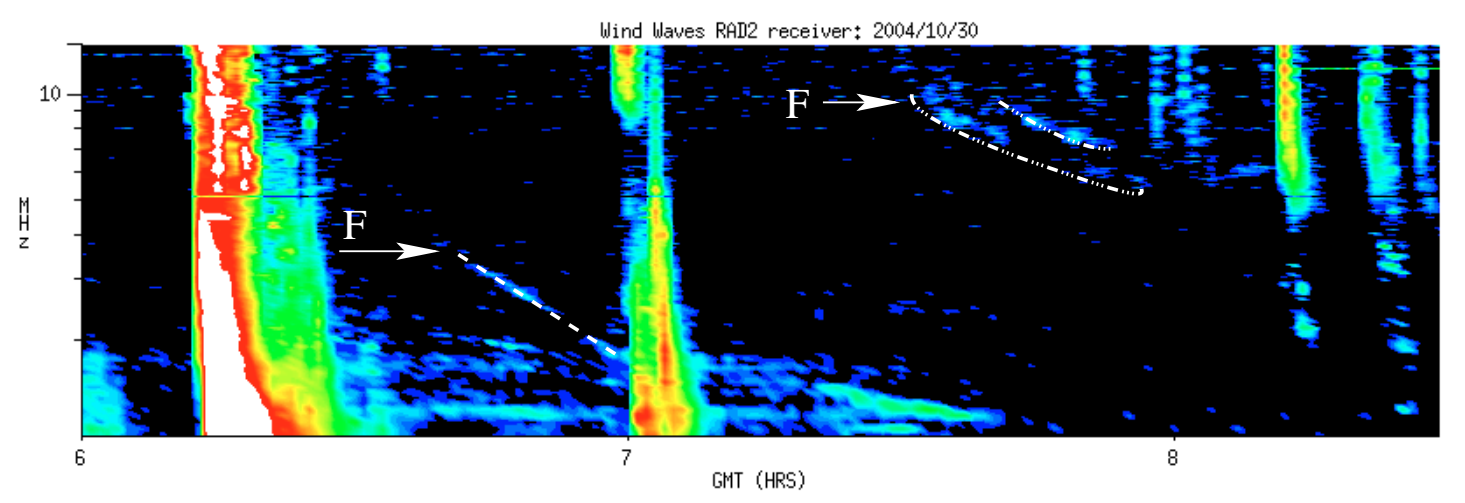

Fig. 8. WIND WAVES RAD2 observations at 1-14 MHz between 06:00 and 08:30 UT. A DH type II burst lane is observed from 06:45 until 07:00 UT (indicated by the dashed line). A pair of faint type II burst lanes is observed later, starting at 07:29 UT around $10 \mathrm{MHz}$ (indicated by the dashed-dotted lines).

through the relation $f=9000 \sqrt{n_{\mathrm{e}}}$. Electron density can then be transformed into atmospheric height using different atmospheric density models. The most widely used density models are by Saito (1970) and Newkirk (1961). We have calculated possible atmospheric height ranges for the type II bursts with a 2-fold Newkirk model (approximation of the active region corona) and with a 2-fold Saito model (approximation for a less dense corona). Frequently-used models like the 4-fold Newkirk and 10-fold Saito would give even larger heights for the burst drivers (see Fig. 8 in Vrs̆nak et al. 2002; Fig. 2 in Warmuth \& Mann 2005; and Fig. 4 in Koutchmy 1994, for the different density models and comparison to observed densities), so our estimates give the reasonable lower limits for the heights. The type II burst times and calculated height ranges are presented in Figs. 9-11, together with other data.

\section{Summary of CME characteristics}

In order to establish the start times of the halo CMEs (defined as the time when the CME would have lifted off from the active region location) we estimated the CME heights from the LASCO C2 images by selecting prominent bright features that could be identified in the next observed $\mathrm{C} 2$ image. The selected radials were shown in Fig. 1. This approach differs from the method used in the LASCO CME Catalogue where only one radial along the leading front is measured, but gives a more reliable approximation for the movement of the whole structure. The height-time plots for each event together with GOES flux curves, type II burst times and heights, and times when EIT dimmings were observed are presented in Figs. 9-11. Our "feature tracking" method can give quite different start times compared to the linear fits on the LASCO CME Catalog leading front locations: for example a linear fit to the first three catalogued (C2) leading front heights of Halo1 gives a start time around 04:30 UT (see the height-times in Fig. 2), the linear fit to our (C2) feature heights gives a start time around 05:30 UT, and a linear fit to the later $(\mathrm{C} 3)$ catalogued leading front heights gives a start time around 06:00 UT.

A further complication is the possibility of CME acceleration at low heights. This cannot be observed directly because of the LASCO C2 field of view limit and the low image

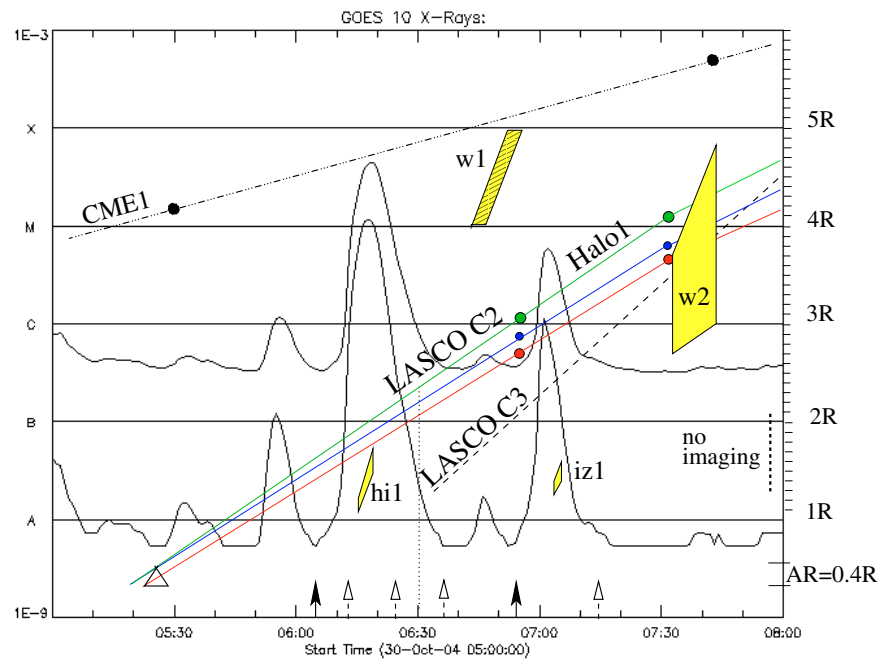

Fig. 9. Estimated heliocentric CME heights in solar radii $(R)$ along the selected radials for the Halo1 event, plotted over the GOES X-ray flux curve. An extrapolated linear fit on the height-times from the later LASCO C 3 observations is also shown. Vertical dotted line shows the time of the earlier $\mathrm{C} 2$ observation when the CME could have been observed if it was within the field of view. The height-times of the preceding CME1 are also shown. Type II burst times and estimated heights (height ranges defined by using 2-fold Newkirk and 2-fold Saito coronal density models) are marked in the plot: "hi1" denotes Hiras observations where fundamental emission starts at 06:15 UT at $150 \mathrm{MHz}$, "iz1" denotes Izmiran observations where fundamental emission starts at 06:42 UT at $90 \mathrm{MHz}$, "w1" denotes WIND RAD2 observations of a DH type II burst starting at 06:42 UT at $3.2 \mathrm{MHz}$, and "w2" denotes a burst starting at 07:32 UT at $10 \mathrm{MHz}$. Triangle indicates the time of an $\mathrm{H} \alpha$ filament eruption around 05:25 UT. The Yunnan observations were limited due to bad weather conditions, but there is some indication of another filament eruption near 06:26 UT. Black arrow shows the start times of two GOES flares at 06:04 and 06:54 UT, and dashed arrows show the times when EIT dimmings were observed (note that there were no available $195 \AA$ EIT images near the start of the second flare, between 06:48 and 07:13 UT). The EIT field of view ends at height $\approx 1.3 R$ and the LASCO C2 field of view begins around $2.1 R$, and thus there is a wide range of heights where imaging is not available. The flaring active region and the dimmed areas were located approximately at $0.4-0.6 R$ on the disk. 


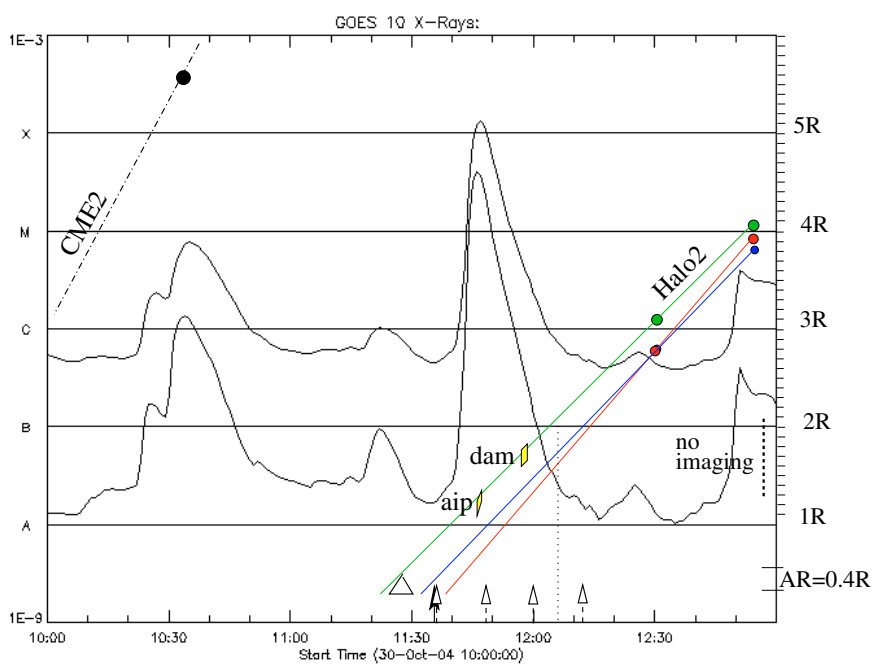

Fig. 10. As in Fig. 9 for the Halo2 event. The type II burst (fundamental emission lane) observed by AIP-Tremsdorf ("aip") starts at 11:45 UT at $140 \mathrm{MHz}$. There is a simultaneous type II burst in the AIP-Tremsdorf dynamic spectra where fundamental emission starts at $80 \mathrm{MHz}$, and the height of this burst exciter would be in between the "aip" and "dam" source heights. "dam" denotes Nançay Decametric Array observations of fundamental emission at $44 \mathrm{MHz}$ starting at 11:58 UT. The height-times of the preceding CME2 are also shown. GOES flare starts at 11:36 UT.

cadence and field of view limit of EIT. The CME leading edge at lower heights corresponds to the coronal structure overlying the erupting filament, see e.g., Maričić et al. (2004, and references therein). Accelerating filaments where the trajectory later matches the CME trajectory have been observed (see, e.g. Figs. 5 and 6 in Qiu et al. 2001). In the cases of observed CME acceleration, it usually occurs within $2-3 R_{\odot}$, during the eruptive phases of associated flares (e.g., Shanmugaraju et al. 2003). It has also been suggested that CMEs can accelarate continuously from 3 to $7 R_{\odot}$ (see Moon et al. 2004 and references therein).

The Halo1 event is the most interesting of the three halo CMEs: the observed (projected) CME speed is low at heights $3-5 R_{\odot}\left(210 \ldots 335 \mathrm{~km} \mathrm{~s}^{-1}\right)$, changing into a higher speed $\left(>400 \mathrm{~km} \mathrm{~s}^{-1}\right)$ after that. There are two flares associated with this event, and the CME velocity change happens one hour after the second flare. Both flares are associated with metric type II burst emission. A CME propagating at a velocity less than $300 \mathrm{~km} \mathrm{~s}^{-1}$ is not likely to produce a shock (as observed at 06:15 UT, marked "hi1" in Fig. 9), or be able to produce another shock ("iz1") at low heights when the CME front is already above $3 \mathrm{R}$. In addition, the two DH type II bursts look like a continuation of the two metric type II bursts. In the Halo2 and Halo3 events the shock signatures in radio match relatively well with the backwards-extrapolated CME feature heights, although Halo2 is slow compared to the Alfvén speed it needs to exceed to produce a shock, see Figs. 10 and 11.

\section{Results and discussion}

We report on flare and CME activity that originated from the same active region and occurred within $12 \mathrm{~h}$ on the same day,

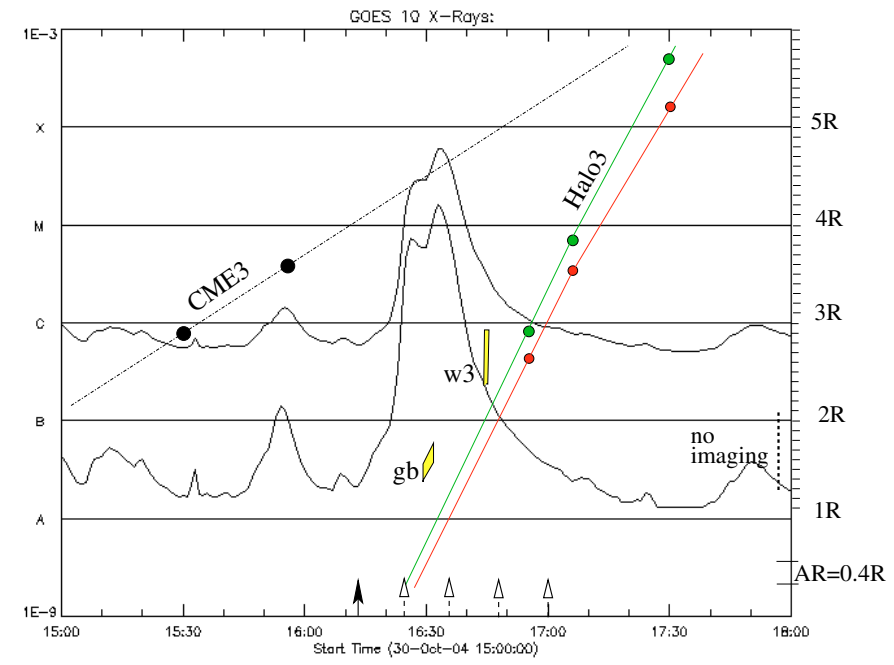

Fig. 11. As in Fig. 9 for the Halo3 event. The type II burst (fundamental emission lane) observed by GBSRBS ("gb") starts at 16:30 UT at $65 \mathrm{MHz}$, and there is a possible continuation in the WIND RAD2 spectrum near 16:44 UT at $14 \mathrm{MHz}$ ("w3"). The height-times of the preceding CME3 are also shown. GOES flare starts at 16:14 UT.

October 30, 2004. The activity included three halo CMEs that were associated with flares and radio type II bursts, as well as one flare and one CME in between the halo CMEs that also showed shock signatures. In addition, all halo-type CME events were associated with similar-looking, large-scale EUV dimmings.

The first two halo CMEs could be called slow if compared to the halo CME speeds on average. The projected leading front speeds were 260 and $325 \mathrm{~km} \mathrm{~s}^{-1}$, respectively, measured from the first two available LASCO images. The third halo CME was fast, $920 \mathrm{~km} \mathrm{~s}^{-1}$. Observations have earlier associated only fast CMEs $\left(>600 \mathrm{~km} \mathrm{~s}^{-1}\right.$ ) with metric type II radio bursts, mainly because the fast ejected mass can act as a driver for the shock-driven radio burst or because fast CMEs are often associated with large impulsive flares that can produce blast waves (a shock is produced when the speed of the disturbance exceeds the local magnetosonic, i.e. Alfvén, speed).

We have analysed the events in order to establish plausible CME lift-off times and to determine the cause of the metric and DH type II bursts that occurred in association with the slow CMEs. First, we used a CME speed measurement technique where distinctive CME front features were identified and height-time positions were measured along several radials. The extrapolated CME start times obtained with this method differ from the ones obtained with the CME Catalog method (measurements of the leading front along one radial). In the case of the first halo CME (Halo1) the difference is significant, giving a one hour time difference. A linear fit to the featuretracked height-time data points gives an estimated CME start time that coincides well with the observed $\mathrm{H} \alpha$ filament eruption. However, we have to take into account the possibility of CME acceleration, although the change from an accelerating filament to a constant velocity CME is not always clear (in the absence of direct observations). 
From the plot in Fig. 9 it is evident that the first metric type II burst associated with Halo1 ("hi1") could have been created by the propagating CME only if the CME started to accelerate near the time of the flare start at 06:04 UT. The speed of Halo1 is low, and the CME would not be able to drive a shock and cause a type II burst in ordinary coronal conditions. An EUV dimming was observed three minutes before the type II burst at loop footpoints in the nearby region, together with loop oscillations/displacements. The association between coronal loop oscillations and metric type II bursts was recently pointed out by Hudson \& Warmuth (2004), and they suggested that the oscillations were the result of weak fast-mode shock waves (flare blast waves) travelling through the corona. The waves could explain both the type II bursts and Moreton waves. We find no observational evidence of Moreton waves in this case, but it can be due to the limited number of observations we have in $\mathrm{H} \alpha$ and in EUV. The DH type II burst ("w1") observed after the metric type II burst suggests that either a blast wave propagated to that distance or the preceding CME1 was able to create the burst. However, CME1 was even slower than Halo1 and therefore not a good candidate to be the burst exciter. CME1 and Halo1 would have reached the same height, 6.5 $R_{\odot}$, around 09:00 UT, and Halo1 had to propagate in the turbulent afterflows of CME1. Hence the coronal densities that were used to determine the type II burst driver heights would need to be considered carefully. There is even one more possibility for the creation of the DH type II burst: Halo1 might have acted as a spherical piston (Landau \& Lifshitz 1987) and in that case even a "sub-sonic" expansion could have created a shock in an analogous way as in the pressure pulse scenario (see Vršnak \& Lulić 2000).

The loop-like front of Halo1 was visible at height $\sim 3 R_{\odot}$ at 06:54 UT, when a C-class flare started in the active region. A metric type II burst "iz1" occurred at 07:03 UT (estimated driver height $1.25-1.4 R_{\odot}$ ), followed by a DH type II burst "w2" at 07:32 UT (estimated driver height 2.7-3.7 $R_{\odot}$ ). A loop displacement/temperature change was observed again, but there was no large-scale dimming in EUV this time. There are three possibilities for the metric type II burst formation in this case: a blast wave shock, shock produced at the flanks of Halo1, or a second mass ejection starting at the time of the flare. A shock at the flanks of the CME is the least probable explanation. Even if shock formation is favoured at the flanks instead of the CME front, CMEs with speeds less than $600 \mathrm{~km} \mathrm{~s}^{-1}$ may not be able to drive shocks around 2.5-3 $R_{\odot}$, where the Alfvén speed has its highest value (Vainio $\&$ Khan 2004). A blast wave scenario is more probable, although the only other indication of it is the observed loop displacement in EUV. The EIT image cadence was very low in this case because of a gap in the $195 \AA$ images between 06:48 and 07:13 UT, and the brightenings/dimmings due to the previous event were still present. The $\mathrm{H} \alpha$ observations were very limited due to bad weather conditions, but the Yunnan $\mathrm{H} \alpha$ images give some indication of structural changes around 06:26 UT near the location where the 05:25 UT filament eruption occurred (see Fig. 3 and the daily full-disk $\mathrm{H} \alpha$ movie from the Global High-resolution H-alpha Network, at ftp://ftp.bbso.njit.edu/pub/archive/2004/10/30).
This third possibility, a later ejection, has support from observations by Moon et al. (2004) where a CME gets accelerated when it is overrun by a faster event associated with a later flare. It is possible that the faster speed of Halo 1 above $5 R_{\odot}$ is connected to a later eruption/ejection, as the $\mathrm{C} 3$ feature heights extrapolated backwards match quite well with the "w2" burst driver heights. A high-speed ejection after Halol could have "pushed" the slow speed Halo1, leaving the first-erupted filament ahead (on top) of the second ejection. This would be a requirement, since the CME front looks essentially the same in the $\mathrm{C} 2$ and $\mathrm{C} 3$ images. However, as there are no large-scale dimmings associated with the second flare, a second large-scale mass depletion is less likely.

Halo2 is also a relatively slow CME that accelerates near $5 R_{\odot}$. The estimated heights of the metric type II bursts, "aip" and "dam" in Fig. 10, fit relatively well with the CME heighttime profiles. If again Halo2 was accelerating at the time of the type II bursts, the shock condition would have been filled. The fast drifts $\left(>2 \mathrm{MHz} \mathrm{s}^{-1}\right)$ observed at the beginning of the burst could also have been caused by a blast wave shock, similar to the event where fast-drift type II-like bursts are in close association with a Moreton wave and an EIT wave (Pohjolainen et al. 2001). There are no DH type II bursts associated with this event, and Halo2 does not seem to have interacted with previous CMEs, although it propagates in the wake of the fast CME2 (that occurred between Halo1 and Halo2). The first signs of loop displacements/dimmings were visible at 11:36 UT, which is the time of the GOES X1.2 flare start and the estimated time of the Halo2 start (linear fit extrapolated backwards). As a filament eruption was observed to be going on before this time, it is evident that a coronal disturbance was already occurring at the time of the flare start.

The third halo event, Halo3, looked similar to the other two halo events in appearance and in loop displacements and large-scale dimmings. Contrary to the other two halos it had a high speed and it seemed to be decelerating after reaching the height of $15 R_{\odot}$. A linear fit to the height-times and extrapolation backwards gives a CME start time well after the flare start. Displaced EUV loops were observed ten minutes after the flare start, and the metric type II burst "gb" (at height 1.37-1.55 $R_{\odot}$ ) and its possible continuation "w3" (2.35-2.95 $\left.R_{\odot}\right)$ agree in timing and height with a coronal disturbance that could have been a flare-related shock wave. A shock wave propagating ahead of Halo3 could explain the height-time differences between the type II bursts and the Halo3 front. Since there must be a certain offset between the burst driver and the bow-shock (Russell \& Mulligan 2002), this type II burst could also have been a CME-related bow shock.

Propagating coronal shocks need driver speeds that exceed the local magnetosonic speed. It does not seem probable that CME fronts with speeds much less than $400 \mathrm{~km} \mathrm{~s}^{-1}$ are capable of driving a shock (type II burst exciter speeds have been estimated to be in the range of $400-2000 \mathrm{~km} \mathrm{~s}^{-1}$ ). In our analysis we have found evidence of flare-related CME acceleration in the cases of two slow halo CMEs. A flare blast wave and a CME bow shock are both good candidates to be the type II burst exciter in the third, fast halo CME event. The repeating flare-halo CMEs and large-scale dimmings also show that the 
field can restore itself in 5-6 h. Our results show that careful analysis is needed in determining event start times and CME speeds, as it is always possible that CMEs accelerate/decelerate or that the CME material originates from more than one eruption. In statistical analysis especially, one should be aware of these complexities.

Acknowledgements. We are grateful to the referee, B. Vršnak, whose suggestions and comments led to a significant improvement of the paper. The Global High Resolution $\mathrm{H} \alpha$ Network is operated by the Big Bear Solar Observatory, New Jersey Institute of Technology, and we are grateful to V. Yurchyshyn for providing the $\mathrm{H} \alpha$ data. In this study we have used radio observations obtained from Hiraiso Solar Observatory (http://sunbase.nict.go.jp/solar/denpa/), ETH Zürich (http://www.astro.phys.ethz.ch/rag/), LaRS (http://helios.izmiran.rssi.ru/lars/LARS.html), Nançay (http://www.obs-nancay.fr/), and Green Bank (http:// www.nrao.edu/astrores/gbsrbs/). We thank H. Aurass for preparing and interpreting the AIP radio spectra. We are grateful to the SOHO EIT, LASCO, and MDI teams for making their data available in their Web archives. The LASCO CME Catalogue is generated and maintained by NASA and the Catholic University of America in co-operation with the Naval Research Laboratory. The catalogue can be accessed at http://cdaw.gsfc.nasa.gov/ CME_list/.

\section{References}

Bougeret, J.-L., Kaiser, M. L., Kellogg, P. J., et al. 1995, Space Sci. Rev., 71, 231

Brueckner, G. E., Howard, R. A., Koomen, M. J., et al. 1995, Sol. Phys., 162, 357

Cane, H. V., \& Erickson, W. C. 2005, ApJ, 623, 1180

Chertok, I. M., Grechnev, V. V., Hudson, H. S., \& Nitta, N. V. 2004, J. Geophys. Res., 109, A02112

Cliver, E. W., Webb, D. F., \& Howard, R. A. 1999, Sol. Phys., 187, 89

Cliver, E. W., Nitta, N. V., Thompson, B. J., \& Zhang, J. 2005, Sol. Phys., 225, 105

Delaboudinière, J.-P., Artzner, G. E., Brunaud, J., et al. 1995, Sol. Phys., 162, 291

Gallagher, P. T., Lawrence, G. R., \& Dennis, B. R. 2003, ApJ, 588, L53

Goff, C. P., van Driel-Gesztelyi, L., Harra, L. K., Matthews, S. A., \& Mandrini, C. H. 2005, A\&A, 434, 761

Gopalswamy, N., Kaiser, M. L., Thompson, B. J., et al. 2000, Geophys. Res. Lett., 27, 1427

Harvey, G. A. 1965, J. Geophys. Res., 70, 2961

Harra, L. K., \& Sterling, A. C. 2001, ApJ, 561, L215

Hudson, H. S., \& Warmuth, A. 2004, ApJ, 614, L85

Hudson, H. S., Khan, J. I., Lemen, J. R., Nitta, N. V., \& Uchida, Y. 2003, Sol. Phys., 212, 121

Jing, J., Yurchyshyn, V. B., Yang, G., Xu, Y., \& Wang, H. 2004, ApJ, 614,1054

Klassen, A., Aurass, H., Klein, K.-L., Hofmann, A., \& Mann, G. 1999, A\&A, 343, 287

Klassen, A., Pohjolainen, S., \& Klein, K.-L. 2003, Sol. Phys., 218, 197

Koutchmy, S. 1994, Adv. Space Res., 14, 429

Kundu, M. R, White, S. M., Garaimov, V. I., et al. 2004, ApJ, 607, 530

Lara, A., Gopalswamy, N., Nunes, S., Muñoz, G., \& Yashiro, S. 2003, Geophys. Res. Lett., 30, SEP 4-1
Landau, L. D., \& Lifshitz, E. M. 1987, Fluid Mechanics (Pergamon Press), 2nd edn.

Mancuso, S., \& Raymond, J. C. 2004, A\&A, 413, 363

Maričić, D., Vršnak, B., Stanger, A. L., \& Veronig, A. 2004, Sol. Phys., 225, 337

Michałek, G., Gopalswamy, N., \& Yashiro, S. 2003, ApJ, 584, 472

Moon, Y. J., Chae, J., Choe, G. S., et al. 2002, ApJ, 574, 1066

Moon, Y. J., Cho, K. S., Smith, Z., et al. 2004, ApJ, 615, 1011

Nelson, G. J., \& Melrose, D. B. 1985, in Solar Radio Physics, ed. D. J. McLean, \& N. R. Labrum (Cambridge: Cambridge Univ. Press), 333

Newkirk, G. Jr. 1961, ApJ, 133, 983

Pohjolainen, S., Maia, D., Pick, M., et al. 2001, ApJ, 556, 421

Pohjolainen, S., Vilmer, N., Khan, J. I., \& Hillaris, A. E. 2005, A\&A, 434, 329

Qiu, J., Wang, H., Cheng, C. Z., \& Gary, D. E. 2001, ApJ, 604, 900

Reiner, M. J., Kaiser, M. L., Gopalswamy, N., et al. 2001, J. Geophys. Res., 106, 25279

Reiner, M. J., Vourlidas, A., St. Cyr, O., et al. 2003, ApJ, 590, 533

Russell, C. T., \& Mulligan, T. 2002, Planetary and Space Sci., 50, 527

Saito, K. 1970, Ann. Tokyo Astr. Obs., 12, 53

Scherrer, P. H., Bogart, R. S., Bush, R. I., et al. 1995, Sol. Phys., 162, 129

Schrijver, C. J., Aschwanden, M. J., \& Title, A. M. 2002, Sol. Phys., 206,69

Shanmugaraju, A., Moon, Y.-J., Dryer, M., \& Umapathy, S. 2003, Sol. Phys., 215, 185

Sheeley, N. R., Walters, J. H., Wang, Y.-M., \& Howard, R. A. 1999, J. Geophys. Res., 104, 24739

Sterling, A. C., \& Moore, R. L. 2005, ApJ, 630, 1148

Vainio, R., \& Khan, J. I. 2004, ApJ, 600, 451

Vršnak, B. 2001a, J. Geophys. Res., 106, 25249

Vrs̆nak, B. 2001b, J. Geophys. Res., 106, 25279

Vršnak, B., \& Lulić, S. 2000, Sol. Phys., 196, 157

Vršnak, B., Ruždjak, V., Zlobec, P., \& Aurass, H. 1995, Sol. Phys., 158,331

Vršnak, B., Magdalenič, J., Aurass, H., \& Mann, G. 2002, A\&A, 396, 673

Vršnak, B., Maričić, D., Stanger, A. L., \& Veronig, A. 2004, Sol. Phys., 225, 355

Vrs̆nak, B., Sudar, D., \& Ruždjak, D. 2005, A\&A, 435, 1149

Warmuth, A., \& Mann, G. 2005, A\&A, 435, 1123

Warmuth, A., Vršnak, B., Magdalenić, J., Hanslmeier, A., \& Otruba, W. 2004a, A\&A, 418, 1101

Warmuth, A., Vršnak, B., Magdalenić, J., Hanslmeier, A., \& Otruba, W. 2004b, A\&A, 418, 1117

Webb, D. F., Cliver, E. W., Crooker, N. U., St. Cyr, O. C., \& Thompson B. J. 2000, J. Geophys. Res., 105, 7491

Wu, S. T., Zhang, T. X., Tandberg-Hanssen, E., et al. 2004, Sol. Phys., 225,157

Yashiro, S., Gopalswamy, N., Michałek, G., et al. 2004, J. Geophys. Res., 109, A07105

Zarro, D. M., Sterling, A. C., Thompson, B. J., Hudson, H. S., \& Nitta, N. 1999, ApJ, 520, L139

Zhang, J., \& Wang, J. 2002, ApJ, 566, L117

Zhang, J., Dere, K. P., Howard, R. A., Kundu, M. R., \& White, S. M. 2001, ApJ, 559, 452

Zhang, J., Dere, K. P., Howard, R. A., \& Vourlidas, A. 2004, ApJ, 604, 420 